\title{
Anwendung von Lernstrategien bei Hochschulstudierenden im Bereich Deutsch
}

\section{Application of learning strategies for university students in the field of German as a foreign language (DaF)}

Martin Lachout, Eva Krovová, Ondřej Moc

\begin{abstract}
The article deals with the question of using learning strategies for DaF in a specific group, namely university students. In particular, we based our research on taxonomies of different learning strategies, and targeted two groups of students: those participating in philological and non-philological study programs. After a questionnaire at Czech universities in 2018, we have statistically analysed and evaluated these strategies. We were particularly interested in whether there are differences in the number of learning strategies used between philologically and non-philologically oriented students.
\end{abstract}

\section{Keywords}

learning strategies, German as a foreign language, strategy research, statistical method 
„It is a tragic fact that most of us only know how to be taught; we haven't learned how to learn."

(KNOWLES 1995: 14)

\section{Einleitung}

Während des Fremdsprachenlernens setzen die Lernenden, bewusst oder auch unbewusst, eine Reihe von Lernstrategien ein, um einerseits das neu Gelernte kognitiv zu verarbeiten, es in ihrem Gedächtnis besser zu speichern und es bei Bedarf einfach abzurufen und andererseits um das eigene Lernen zu planen, zu überwachen, zu reflektieren und auszuwerten. Diese Verhaltensweisen, die sog. Lernstrategien, helfen den Lernenden, ihr Ziel zu erreichen - ihre kommunikative Kompetenz in einer Fremdsprache zu entwickeln. Die Lernstrategienforschung hat eine relativ lange Tradition, die in die 70er Jahre des 20. Jahrhunderts reicht. Die ersten Klassifizierungen (Taxonomien) sind schon in dieser Zeit als Ergebnis der Lernstrategienforschung bei erfolgreichen Lernenden entstanden (z. B. Rubin 1975 in Westhoff 2001: 685, Ellis 1994). Westhoff und Ellis selbst widmen sich den Lernstrategien in Hinsicht auf das Fremdsprachenlernen. Laut Streblow/Schiefele (2006: 352) lieferten wichtige Befunde zu Lernstrategien in den 70er Jahren die Arbeitsgruppen von Marton (vgl. Marton/Säljö 1976: 4-11) und Entwistle (vgl. Entwistle/Ramsden 1983: 6-28). Nach dem Jahr 2000 führten mehrere Autoren (z. B. Schiefele et al. 2003, Jamieson-Noel/Winne 2003) Studien durch, die sich Lernstrategien und ihrer Effekte auf Lernleistungen widmeten.

Lernstrategien im Studium im Allgemeinem widmet sich Wild in seinem Buch, der sich mit der Frage befasst, wie die Struktur von Lernstrategien im Studium in geeigneter Weise definiert und gemessen werden kann und welche individuellen und situativen Bedingungen für die Nutzung kognitiver Lernstrategien verantwortlich sind (vgl. Wild 2000: 84-114).

Mit charakteristischen Merkmalen von Lernstrategien, konkret auf die Lernstrategien beim Fremdsprachenlernen, befasste sich in den 90er Jahren auch Oxford (1990), die als Bahnbrecherin auf diesem Forschungsfeld gilt und an die unseres Erachtens andere Autoren anknüpfen.

Im tschechischen Kontext handelt es sich um einen ziemlich jungen Forschungsbereich der Fremdsprachendidaktik. Intensiv haben sich mit diesem Thema z. B. Vlčková (2007), Janíková (2007) oder Lojová/Vlčková (2011) befasst. Die vorliegende Studie versteht sich in diesem Zusammenhang als eine Fortsetzung dieser Forschungen im universitären Bereich. Durch eine breit angelegte Befragung der DaF-Lernenden an tschechischen Universitäten sollte festgestellt werden, welche Lernstrategien diese beim Deutschlernen/-studieren einsetzen und ob es dabei erhebliche Unterschiede zwischen den Studierenden der Germanistik (d. h. den Philologen) und Studierenden anderer Studienprogramme, die jedoch auch Deutsch lernen, gibt. 


\section{Das Wesen der Lernstrategien}

Lernstrategien eindeutig zu definieren ist nicht einfach, denn das Konzept der Lernstrategien kann aus verschiedenen Perspektiven betrachtet werden. Für unsere Zwecke sind die Perspektive der Kognitionswissenschaften und die der Fremdsprachendidaktik von Bedeutung, die einen wichtigen Ansatz für die von uns untersuchte Problematik bilden.

Es handelt sich bei Lernstrategien um „Handlungssequenzen zur Erreichung eines Lernziels“ (nach Friedrich/Mandl 1992: 6), um „Entscheidungsregeln bzw. Handlungsprogramme“ (nach Lompscher 1996: 237), „Bestandteile eines Planungsprozesses“ (nach Færch /Kasper 1983: 215), um „zum Lernen bzw. zur Regulierung des Lernprozesses eingesetzte Verhaltensweisen“ (nach Wenden 1987: 6) oder „Aktionen (nach Oxford 1990: 11) und Gedanken mit dem Fokus auf der Verarbeitung von Informationen“ (nach O’Malley/Chamot 1990: 43), um „vom Lerner eingesetzte Operationen, die beim Erwerb, der Speicherung und dem Abrufen von Informationen helfen“ (nach Oxford 1990: 15) oder um „eine Form des Wissens, genaues Wissen über das Lernen und die Strategien, die benutzt werden“ (nach Wenden 1987: 9).

Cohen fasst die oben genannten Definitionen zusammen, wenn er sagt, dass die Sprachlern- und Sprachgebrauchsstrategien als Prozesse definiert werden können, die die Schüler bewusst wählen, um das Lernen oder den Gebrauch einer zweiten Sprache oder einer Fremdsprache zu verbessern, indem die Informationen über diese Sprache(n) gespeichert, erhalten, abgerufen und angewendet werden (vgl. Cohen 2014: 4).

Passend definieren die Lernstrategien in ihrem Aufsatz Streblow und Schifele, nach denen es sich „bei Lernstrategien (a) um eine Abfolge von effizienten Lerntechniken handelt, die (b) zielführend und flexibel eingesetzt werden, (c) zunehmend automatisiert ablaufen, aber (d) bewusstseinfähig bleiben“ (Streblow/Schiefele 2006: 353).

Die Lernstrategien weisen auch gewisse charakteristische Merkmale auf, die z. B. Oxford (1990: 13) treffend nennt:

- die Lernstrategien tragen generell zum Hauptziel bei dem Erwerb von kommunikativer Kompetenz bei,

- $\quad$ sie bieten den Lernenden die Möglichkeit ihren eigenen Lernprozess selbst zu leiten,

- sie sind problemorientiert,

- $\quad$ es handelt sich um spezifische, vom Schüler gewählte Schritte zur Aneignung der Fremdsprache,

- $\quad$ sie umfassen nicht nur die kognitive Seite, sondern auch viele andere Aspekte der Lernenden (z. B. soziale),

- $\quad$ sie fordern das Lernen sowohl direkt als auch indirekt,

- sie sind nicht immer beobachtbar,

- $\quad$ in den meisten Fällen werden sie von Studierenden absichtlich eingesetzt,

- sie sind erlernbar, aber

- $\quad$ sie werden durch eine Reihe von verschiedenen Faktoren beeinflusst. 
Im Zusammenhang mit den genannten charakteristischen Merkmalen von Lernstrategien ergibt sich zwingend auch die Frage nach der Bewusstheit bei deren Anwendung, die jedoch nicht eindeutig beatwortet werden kann. Færch und Kasper haben festgestellt, dass die Bewusstheit als schrittweise bewusste bzw. unbewusste Anwendung von Lernstrategien zu verstehen sei. Dies sei von der Individualität jedes Lernenden abhängig, von der Art und Weise, wie er oder sie beim Lernen vorgeht. Eine anfangs bewusst applizierte Strategie kann mit der Zeit dank der häufigen Anwendung automatisiert werden, sodass der Lernende sich des Einsatzes nicht mehr bewusst sei (vgl. Færch / Kasper 1983: 324).

Laut der deutschen Autorin Rampillon versteht man unter Lerntechniken und Lernstrategien strategische Verhaltensweisen, die die Lernenden bei der Aneignung und Verarbeitung neuer Informationen anwenden (vgl. Rampillon 1985: 13).

Wir betrachten Lernstrategien als solche Verfahren, die das Lernen verbessern und beschleunigen und die zugleich Lernenden/Studierenden helfen, entstandene Probleme effektiv zu bewältigen und Aufgaben auf solch eine Art zu lösen, die der Situation oder dem Kontext entspricht. Je mehr Strategien Lernende/Studierende beherrschen, desto höher ist ihre Chance auf Erfolg beim Problemlösen.

\subsection{Lernstrategien aus der Sicht der kognitiven Psychologie}

Wie schon aufgeführt, sind Lernstrategien eng mit der Kognition des Lernenden verbunden, d. h. mit der Art und Weise seiner Wahrnehmung, Informationsverarbeitung, Gedächtnisfunktionsfähigkeit, Problemlösungsfähigkeit etc. Aus dem Grund widmen wir an dieser Stelle unsere Aufmerksamkeit den Lernstrategien aus kognitiver Sicht.

Der Ausgangspunkt der Kognitionswissenschaft ist die komplexe Informationsverarbeitung beim Lernen, wobei dieser Prozess seitens des Lernenden selbst konzipiert ist (vgl. z. B. Weinstein/Mayer 1986: 320). Der Lernende muss die Information zuerst geistig verarbeiten, damit er sie anschließend in sein schon vorhandenes Wissen einordnen kann. Dieser Prozess ist nur dann möglich, wenn beim Lernenden die kognitiven Vorgänge einwandfrei funktionieren und die Teiloperationen fehlerfrei realisiert werden.

Lernstrategien stellen für die kognitive Psychologie ein interessantes Forschungsfeld dar, denn es wird vorausgesetzt, dass ihre Anwendung von unterschiedlichsten kognitiven Aktivitäten beeinflusst wird. Die Beziehung zwischen den kognitiven Prozessen, Operationen und Lernstrategien kann wie folgt formuliert werden: Die Lerntätigkeit führt zur Erweiterung und Umstrukturierung von Wissen mithilfe kognitiver Prozesse und Operationen. Lernstrategien tragen zur Systematisierung des Lernprozesses bei. Sie werden somit als die kleinsten Bestandteile der kognitiven Prozesse wahrgenommen.

\subsection{Lernstrategien aus der Sicht der Fremdsprachendidaktik}

Die Schlüsselfrage der Fremdsprachendidaktik ist, wie sich erreichen lässt, dass die Lerner möglichst schnell und effizient lernen und dabei fähig sind, ihr gewonnenes Wissen und 
ihre Kenntnisse später effektiv umzuwandeln, im Falle einer Fremdsprache heißt das: in einer realen kommunikativen Situation anwenden zu können. Wichtig ist dabei, wie der Lernende vorgeht, mit welchen Lernverfahren er im Unterricht konfrontiert wird, ob diese in seinem Fall effektiv sind oder nicht. Rampillon (1985: 215) z. B. versteht in diesem Zusammenhang Lernstrategien als Verhaltensweisen oder als Arbeitsweisen der Lernenden.

Wie schon erwähnt, fallen die ersten Forschungen zu Lernstrategien hinsichtlich des Fremdsprachenlernens bzw. (auch) -lehrens in die 70er Jahre des 20. Jahrhunderts. Im deutschsprachigen Kontext hat Rampillon die Notwendigkeit der Vermittlung von Lernstrategien im Fremdsprachenunterricht betont. Im Vergleich zur oben aufgeführten Interpretation von Rampillon (1985: 215) bringt sie später gemeinsam mit Bimmel eine erweiterte und ergänzte Betrachtungsweise auf Lernstrategien, diesmal schon auch in Bezug auf Lehren. Gemäß diesen Autoren ist

eine Lernstrategie [...] ein Plan, den jemand im Kopf hat, um ein Ziel zu erreichen. Fremdsprachenlernende wenden sie an, um eine Fremdsprache möglichst erfolgreich zu lernen: Lernstrategien sind ein Plan (mentalen) Handelns, um ein Lernziel zu erreichen. Um sich eine Lernstrategie zurecht legen zu können, muss den Lernenden das Lernziel klar sein. Das lässt sich gut in einer „Wenn...dann-Formulierung“ ausdrücken: Wenn es mein Ziel ist, die Bedeutung eines Wortes zu ermitteln, dann schlage ich das Wort im Wörterbuch nach. (Bimmel/ Rampillon 2000: 196)

Die bei der Anwendung der Sprache eingesetzten Strategien werden von Bimmel und Rampillon wie folgt definiert:

Strategien, die nicht wie die eigentlichen Lernstrategien so sehr für das Erlernen, sondern eher für den kommunikativen Gebrauch und das Verstehen der Fremdsprache verwendet werden. Dazu gehören auch alle Möglichkeiten, eine Kommunikation aufrechtzuerhalten, wie etwa Gestik, Mimik, Umschreibungen von Wörtern usw. Sie dienen also der Kompensation, um Mängel in der Beherrschung der Fremdsprache auszugleichen. (Bimmel/Rampillon 2000: 197)

Nach dieser Auffassung soll sich der Lerner dessen bewusst werden, was für sein möglichst effektives Fremdsprachenlernen, bzw. Sprachlernen zu tun ist. Andererseits darf nicht vergessen werden, dass die Anwendung von Lernstrategien selbst noch keine Garantie für einen problemlosen Lernprozess ist. Dieser hängt von vielen Variablen ab, wie z. B. von der Motivation, vom Stand der Iterimsprache, der Anwendung der ausgewählten Lernstrategie u. Ä. Im Vordergrund steht dann die Frage, wie oder warum sich der Lernende entscheidet, ob die von ihm gewählte Lernstrategie richtig ist oder nicht. Dabei können folgende Situationen eine Rolle spielen:

- Der Lernende probiert beim Problemlösen verschiedene alternative Strategien und wertet ihre Effizienz aus.

- Der Lernende tauscht seine Erfahrungen mit anderen Lernenden aus und macht auf die Strategien aufmerksam, die er im Hinblick auf das Erreichen des Ziels selbst für am effektivsten hält. 
- Der Lernende entwickelt seine eigenen Hypothesen über effektive Strategien (oder sollte diese entwickeln).

An dieser Stelle ist es auch in Bezug auf die eigene im Text vorgestellte Forschung wichtig, sich die Frage zu stellen, welche Faktoren den Einsatz von Lernstrategien beim Fremdsprachenlernen und -lehren unterstützen. Nach Streblow und Schiefele sind die wichtigsten der bislang untersuchten Bedingungsfaktoren das Vorwissen, Studieninteresse und intrinsische Motivation, extrinsische Lernmotivation, Lehrqualität, Lehrkonzeption der Lehrenden, epistemologische Überzeugungen, Fachrichtung und Studiendauer (Streblow/ Schiefele 2006: 355).

\section{Klassifizierungen der Lernstrategien beim Fremdsprachenlernen}

Es gibt mehrere, auf verschiedenen Kriterien basierende Klassifizierungen von Lernstrategien, so z. B. die auf psychologischen Funktionen basierende Klassifizierung von O'Malley/Chamot (1990: 44), die drei Typen von Lernstrategien unterscheiden: 1. kognitive, 2. metakognitive und 3. sozialaffektive.

Weiter die auf der Linguistik basierende Klassifizierungen mit Fokus auf die Problematik der Bedeutungserschließung, des Sprachmonitorings (vgl. Bialystok 1981: 24-35) oder der Kommunikationsstrategien, wie z. B. Paraphrasieren (vgl. Tarone 1983), oder die auf Sprachfertigkeiten basierende Klassifizierung von Cohen (1990) u. a.

Auf der Klassifizierung von O'Malley/Chamot (1990) basieren auch zwei andere Klassifizierungen, die sich in neueren Forschungen terminologisch durchgesetzt haben. Im deutschsprachigen Kontext ist es die Klassifizierung von Bimmel/Rampillon (2000), im nordamerikanischen Kontext die von Oxford (1990).

Die schon mehrmals erwähnten Autoren Bimmel und Rampillon teilen die Lernstrategien in zwei große Gruppen ein, in die sog. direkten (kognitiven) Strategien (Gedächtnisstrategien und Sprachverarbeitungsstrategien) und in die sog. indirekten Strategien (Strategien zur Regulierung des eigenen Lernens, affektive Strategien, soziale Strategien und Sprachgebrauchsstrategien). Dabei gehen sie von der Klassifizierung von Oxford (1990) aus. Aus Platzgründen können wir uns nicht allen Klassifizierungen detailliert widmen, da jedoch die Klassifizierung der Lernstrategien von Oxford (1990) die komplexeste ist und am besten unserem Forschungsanliegen entspricht, führen wir sie an dieser Stelle an.

\subsection{Klassifizierung von Lernstrategien bei Oxford}

Oxford unterscheidet zwei Gruppen von Lernstrategien - die direkten und die indirekten (vgl. Oxford 1990: 16).

Die direkten Strategien definiert sie folgendermaßen: 
$[\ldots]$ language learning strategies that directly involve the target language [...] All direct strategies require mental processing of the language, but the tree groups of direct strategies (memory, cognitive and compensation) do this processing differently and for different purposes Memory strategies, such as grouping or using imagery, have a highly specific function: helping students store and retrieve new information. Cognitive strategies, such as summarizing or reasoning deductively, enable learners to understand and produce new language by many different means. Compensations strategies, like guessing or using synonyms, allow learners to use the language despite their often-large gaps in knowledge. (Oxford 1990: 37)

Die direkten Strategien tragen unmittelbar zum Fremdsprachenlernen bei. Oxford unterteilt die direkten Strategien ihrerseits in

1. Gedächtnisstrategien, die dem Lerner beim Speichern und Aufrufen neuer Informationen helfen, wie z.B. Visualisierungen, Verbinden von Informationen, strukturiertes Wiederholen etc.,

2. kognitive Lernstrategien, die die Rezeption und die Produktion der neuen Sprache mit Hilfe verschiedener Mittel wie Deduktion, Analyse, Zusammenfassung etc. ermöglichen,

3. Kompensationsstrategien, die dem Lernenden beim Überwinden seiner sprachlichen Defizite in der Kommunikation helfen, z.B. Anwendung von Synonymen, Paraphrasieren etc. (vgl. Oxford 1990: 16).

Die indirekten Strategien dienen dem Lernmanagement, einfacher gesagt, sie erleichtern das Fremdsprachenlernen indirekt. Es handelt sich um:

1. metakognitive Strategien, die den Lernenden eine Kontrolle über ihre Kognition und Koordinierung ihres eigenen Lernens ermöglichen. Es handelt sich dabei um die Zielsetzung, Planung, Vorbereitung, das Monitoring und die Evaluation eigenen Lernens, das Organisieren von Lernmaterialien etc.,

2. affektive Strategien, die den Lernenden helfen, Emotionen zu kontrollieren, wie Angst, Freude etc.,

3. Sozialstrategien ${ }^{1}$, die das Lernen mittels der Interaktion mit anderen fördern, wie z. B. Nachfrageaktivitäten, Kooperationsbereitschaft, Entwicklung von Empathie etc. (vgl. Oxford 1990: 135).

Von dieser Klassifizierung gehen später in ihren Arbeiten z. B. Bimmel/Rampillon (2000: 65-66) aus. Sie unterscheiden:

\section{Direkte (kognitive) Strategien}

a) Gedächtnisstrategien

b) Sprachverarbeitungsstrategien

1 Auch diese Strategien spielen im Lernprozess eine wichtige Rolle, denn der Sprachgebrauch stellt einen sozialen Akt dar. Darüber hinaus verläuft auch Lernen in den meisten Fällen im Rahmen der Interaktion mit anderen Menschen. 


\section{Indirekte Strategien}

a) Strategien zur Regulierung des eigenen Lernens

b) affektive Strategien

c) soziale Strategien

d) Sprachgebrauchsstrategien

Diese Klassifizierung ist auch für unsere Forschung relevant.

\section{Eigene Forschung zur Anwendung von Lernstrategien}

\subsection{Das Ziel der Forschung und Forschungshypothesen}

Das Anliegen unserer eigenen qualitativ-quantitativen Forschung war es, anhand einer 2018 an tschechischen Universitäten und Hochschulen durchgeführten Fragebogenerhebung Lernstrategien bei zwei Gruppen von Studierenden mit Hilfe von statistischen Methoden zu analysieren und anschließend auszuwerten. Es handelte sich dabei um Studierende der philologisch orientierten Studienprogramme und um Studierende, die nicht-philologisch ausgerichtete Programme, wie z. B. BWL, Internationale Beziehungen, Medienwissenschaften u. a., studieren.

Es interessierte uns zugleich, welche der festgestellten Strategien bei Philologen und welche bei Nicht-Philologen (siehe oben) dominieren und ob generell gravierende Unterschiede zwischen den beiden Gruppen von Testpersonen festzustellen sind.

Vor der Datenerhebung wurden 4 Hypothesen ${ }^{2}$ formuliert. In diesem Beitrag wird jedoch aus des für diesen Beitrag festgesetzten Umfangs lediglich auf die ersten beiden Hypothesen näher eingegangen (s. Abschnitt 4.4).

\subsection{Probanden und Datenerhebung}

An der Befragung und somit Datenerhebung nahmen 257 Studierende des ersten Studienjahrs und 108 Studierende des 3. Studienjahrs (Bachelorstudiengänge) von insgesamt 14 tschechischen Universitäten und Hochschulen (davon 71,95 \% Frauen und 28,01\% Männer) teil. Von den insgesamt 356 Befragten waren 58 Studierende von philologisch ausgerichteten Studienprogrammen - deutsche Philologie - (d. h. 16,29 \%). Die Befragung

2 Hypothese Nr. 1: Studierende philologisch orientierter Studienfächer wenden mehrere Lernstrategien beim Deutschlernen an im Vergleich mit Studierenden nicht-philologisch orientierter Studienfächer.

Hypothese Nr. 2: Es gibt gravierende Unterschiede bei den angewandten Lernstrategien zwischen Studierenden der philologisch orientierten und nicht-philologisch orientierten Studienfächer.

Hypothese Nr. 3: Metakognitive Strategien werden von Studierenden häufiger angewandt, als Gedächtnisstrategien oder kognitive Strategien.

Hypothese Nr. 4: Studierende mit einer besseren Kenntnis der deutschen Sprache nutzen metakognitive Strategien in einem höheren Maße als Studierende, deren Kenntnis des Deutschen nicht so entwickelt ist. 
verlief elektronisch, und es sollte festgestellt werden, welche Motivation Studierende zum DaF-Studieren/Lernen haben und welche Lernstrategien sie anwenden. Der Fragebogen $^{3}$ bestand aus drei Teilen (34 Hauptfragen). Im ersten Teil wurden die notwendigen Kontextinformationen erfragt (Studiengang, Studienjahr, Geschlecht, Universität etc.), Informationen über die erlernten bzw. studierten Fremdsprachen (L1, L2 bis Lx) sowie über das selbstempfundene Niveau nach dem Gemeinsamen europäischen Referenzrahmen für Sprachen und Informationen über die Erfahrung mit DaF (Schule, Studium, ERASMUS-Aufenthalt, andere Kontexte). Der zweite Teil befasste sich mit der Motivation für Deutschlernen/-studieren der Studierenden und der dritte Teil, konkret die Hauptfragen 27-34, mit Lernstrategien (nach Bimmel und Rampillon, 2000: 65-66), die mithilfe von insgesamt 69 Teilfragen festgestellt wurden. Die Daten wurden dann mittels statistischer Datenverarbeitung analysiert. Im Folgenden werden einige Analyseergebnisse vorgestellt.

\subsection{Methodologie der Datenverarbeitung}

Bei der statistischen Verarbeitung der erhobenen Daten wurde die Normalität der kontinuierlichen Variablen in betrachteten Dateien mit Hilfe des Kolmogorov-Smirnov-Tests, resp. Shapiro-Wilkov-Tests (např. Netolická, 2008), überprüft. Bei Bedarf wurde die Übereinstimmung der Streuung der entsprechenden Variablen mit Hilfe des Levene-Tests verifiziert. Für den Vergleich der Stufen der einzelnen Variablen wurde der t-Test im Falle einer nicht widerlegten Normalität der analysierten Daten angewandt. Im Falle einer Widerlegung der Normalitätsvoraussetzung der Daten wurden nichtparametrische Tests angewandt - bei abhängigen Daten der Zeichentest von Wilcoxon, bei unabhängigen Daten wurde zu ihrem Vergleich der Mann-Whitney-Test angewandt. Alle im Text erwähnten Tests wurden mittels statistischen SW IBM SPSS ${ }^{4}$ durchgeführt und, falls im Text nicht anders angeführt, lagen auf dem $5 \%$ Signifikanzniveau.

\subsection{Präsentation und Interpretation der Teilergebnisse}

An dieser Stelle versuchen wir die ersten beiden der 4 aufgestellten Hypothesen zu verifizieren oder auch zu falsifiziren.

Hypothese Nr. 1: Studierende philologisch orientierter Studienfächer wenden mehrere Lernstrategien beim Deutschlernen an im Vergleich mit Studierenden nicht-philologisch orientierter Studienfächer.

3 Aus Gründen des für diesen Beitrag festgelegten Umfangs war es nicht möglich hier die ganze Fassung des Fragenbogens zu präsentieren. Der komplette Fragenbogen ist für Interessierte unter dieser Adresse zu finden: https://docs.google.com/forms/d/1sGV0NXU10h1MzE49K82s3hxp4buQ3ArYblQH4s16NW0/ edit?usp=drive_web [20.10.2019]

4 Statistische Software IBM SPSS, Version 25. IBM SPSS, eines der meist verwendbaren Computerprogramme zur statistischen Datenverarbeitung. 
Für die Überprüfung dieser Hypothese wurde eine neue statistische Variable eingeführt, die die Zahl der vom Probanden angewandten Lernstrategien zeigt. Aus diesem Grund war es nötig, ein Kriterium festzusetzen, nach dem bestimmt wird, ob der jeweilige Proband die entsprechende Lernstrategie anwendet oder nicht. Zu diesem Zweck wurde für jede entsprechende Lernstrategie ein Durchschnitt aus allen Antworten errechnet. Falls dieser Durchschnitt einen Wert von mindestens 3,1 erreichte, wurde für den entsprechenden Probanden die Lernstrategie als ,angewandt' angesehen. Dieses Verfahren wurde auch bei anderen Lernstrategien wiederholt.

Anschließend wurde nach dem angeführten Kriterium für jeden Probanden eine Zahl der angewandten Strategien bestimmt, dank derer wir eine neue Variable gewonnen haben, die die Zahl der angewandten Strategien für jeden Probanden bestimmte. Die Gesamtheit der Probanden wurde anschließend in zwei Gruppen geteilt, je nach Zugehörigkeit zur Gruppe der Studierenden eines philologisch orientierten resp. eines nicht philologisch orientierten Studienprogramms (im Weiteren Philologen resp. NichtPhilologen). Die Grafiken 1 und 2 visualisieren die Verteilung der Zahl angewandter Strategien für die Gruppe der Philologen und Nicht-Philologen. Die angeführten Historiogramme weisen unterschiedliche Schiefen auf. Bei Philologen ist die Schiefe negativ $(s=-0,272)$, bei Nicht-Philologen ist sie positiv $(s=0,357)$. Dieser Unterschied in der Schiefe deutet darauf hin, dass die Gruppe der Philologen durchschnittlich eine höhere Zahl an Strategien anwendet.

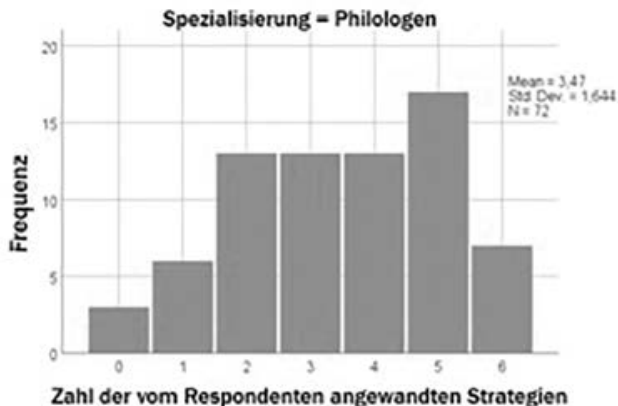

Graphik 1: Zahl der angewandten Strategien in der Philologen-Gruppe

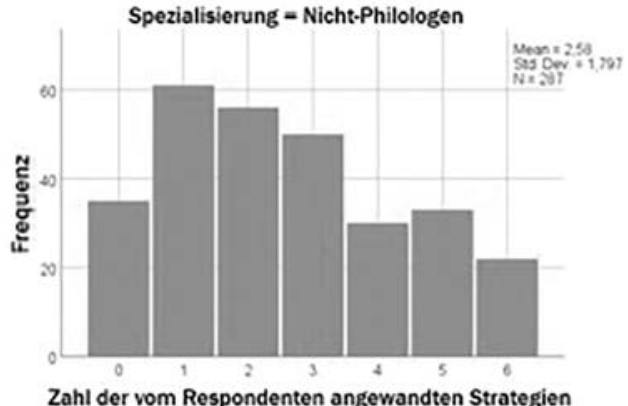

Graphik 2: Zahl der angewandten Strategien in der Nicht-Philologen-Gruppe

Mit Kolmogorov-Smirnov-Tests resp. Shapiro-Wilkov-Tests wurde überprüft, ob dieser Unterschied statistisch signifikant ist. Sowohl für die Philologen als auch für die Nicht-Philologen wurde die Datennormalität mithilfe der beiden o.a. Tests festgestellt. Die Testergebnisse zeigt Tabelle 1 . Alle $p$-Werte waren kleiner als 0,01 , das bedeutet, dass wir die Hypothese über eine Normalverteilung der Zahl angewandter Lernstrategien für die Gruppe der Philologen und Nicht-Philologen widerlegen. 


\begin{tabular}{|c|l|c|c|}
\hline \multicolumn{4}{|c|}{ Normalitätstest } \\
\hline & Spezialisierung & $\begin{array}{c}\text { Kolmogorov- } \\
\text { Smirnov }\end{array}$ & Shapiro-Wilk \\
\hline \multirow{2}{*}{$\begin{array}{c}\text { Zahl der vom Probanden } \\
\text { angewandten Strategien }\end{array}$} & Philologen &, 000 &, 000 \\
\cline { 2 - 4 } & Nicht-Phil. &, 000 &, 001 \\
\hline
\end{tabular}

Tabelle 1: Normalitätstest für die Einteilung des Wertes „Zahl der angewandten Strategien in Hinsicht auf die Spezialisierung"

In Hinsicht auf die Widerlegung unserer Hypothese der Zahlnormalität von angewandten Lernstrategien wurde für die Komparation des Niveaus der Lernstrategienzahl zwischen Philologen und Nicht-Philologen der Mann-Whitney-Test angewandt. Die Testergebnisse sind in Tabelle 2 angeführt.

\begin{tabular}{|lc|}
\hline \multicolumn{2}{|c|}{ Teststatistiken } \\
\hline Mann-Whitney U & 7328,000 \\
Z & $-3,864$ \\
Asymp. Sig. (2-tailed) &, 000 \\
\hline
\end{tabular}

Tabelle 2: Ergebnis des Mann-Whitney-Tests für den Niveauvergleich der Zahl von Lernstrategien zwischen der Philologen-Gruppe und Nicht-Philologen-Gruppe

Der p-Wert des Tests ist auf den ersten drei Dezimalzahlen gleich Null, deshalb ist die Hypothese über die Gleichheit der Verteilung von Lernstrategien in beiden Gruppen widerlegt.

Ergebnis: Die Zahl der von Philologen angewandten Lernstrategien ist höher als die Zahl der Strategien bei Nicht-Philologen.

Hypothese Nr. 2: Es gibt gravierende Unterschiede bei den angewandten Lernstrategien zwischen Studierenden der philologisch orientierten und nicht-philologisch orientierten Studienfächer.

In diesem Teil unserer Forschung konzentrierten wir uns auf die Komparation der Unterschiede bei der Anwendung von einzelnen Kategorien der Lernstrategien bei Philologen und bei Nicht-Philologen. Schrittweise wurden die einzelnen Lernstrategien betrachtet, und es wurde überprüft, ob die Anwendungsunterschiede seitens beider Probandengruppen statistisch signifikant sind. Zu diesem Zweck wurden neue Variablen eingeführt, von denen jede einzelne dem Durchschnitt aller Antworten, die auf die jeweilige Lernstrategie bezogen sind, gleich ist. Dadurch entstanden für jeden Probanden sechs neue Variablen.

Vor der eigentlichen Testdurchführung war es nötig, die Datennormalität über eine durchschnittliche Auswertung der Lernstrategien in der Philologen- und Nicht-Philologen-Gruppe zu überprüfen. In Tabelle 3 sind die ausgerechneten $p$-Werte für einzelne Fälle aufgeführt. 


\begin{tabular}{|l|c|c|c|c|}
\hline \multirow{2}{*}{ Teste der Datennormalität } & \multicolumn{2}{|c|}{ Kolmogorov-Smirnov } & \multicolumn{2}{c|}{ Shapiro-Wilk } \\
\cline { 2 - 5 } & Nicht-Phil. & Philologen & Nicht-Phil. & Philologen \\
\hline$\varnothing^{5}$ - Gedächtnisstrategien & 0,009 & $0,200^{*}$ & 0,040 & 0,334 \\
\hline$\varnothing$ - Kognitive Strategien & 0,004 & 0,022 & 0,050 & 0,003 \\
\hline$\varnothing$ - Kompensationsstrategien & 0,022 & $0,200^{*}$ & 0,031 & 0,686 \\
\hline$\varnothing$ - Metakognitive Strategien & 0,003 & $0,200^{*}$ & 0,580 & 0,058 \\
\hline$\varnothing$ - Affektive Strategien & 0,002 & $0,200^{*}$ & 0,000 & 0,170 \\
\hline$\varnothing$ - Soziale Strategien & 0,008 & $0,200^{*}$ & 0,011 & 0,181 \\
\hline
\end{tabular}

Tabelle 2: Normalitätsüberprüfung für die durchschnittliche Anwendung von Lernstrategien

Aus der Tabelle geht hervor, dass für die Nicht-Philologen-Gruppe immer wenigstens ein p-Wert (für den Kolmogorov Smirnov-Test oder den Shapiro-Wilk-Test) kleiner als 0,05 ist. Deshalb ist auf dem $5 \%$-Signifikanzniveau die Hypothese einer normalen Durchschnittsverteilung aller Lernstrategien bei der Nicht-Philologen-Gruppe widerlegt. Für die Philologen-Gruppe ergaben sich in den beiden Normalitätstests $p$-Werte, die höher als 0,05 bei allen getesteten Variablen waren, außer bei den kognitiven Strategien. Deshalb ist die Hypothese einer normalen Durchschnittsverteilung bei der Philologen-Gruppe nur für kognitive Strategien ebenfalls widerlegt.

Zur Bestimmung der Unterschiede in der Anwendung einzelner Strategien in Hinsicht auf die Zugehörigkeit zur Philologen-Gruppe oder Nicht-Philologen-Gruppe wurde von der Graphik Nr. 3 ausgegangen. In der dargestellten Graphik sind die Box-Plots markiert, die die Durchschnittswerte einzelner Lernstrategien bei einzelnen Probanden der Philologen-Gruppe und Nicht-Philologen-Gruppe darstellen.

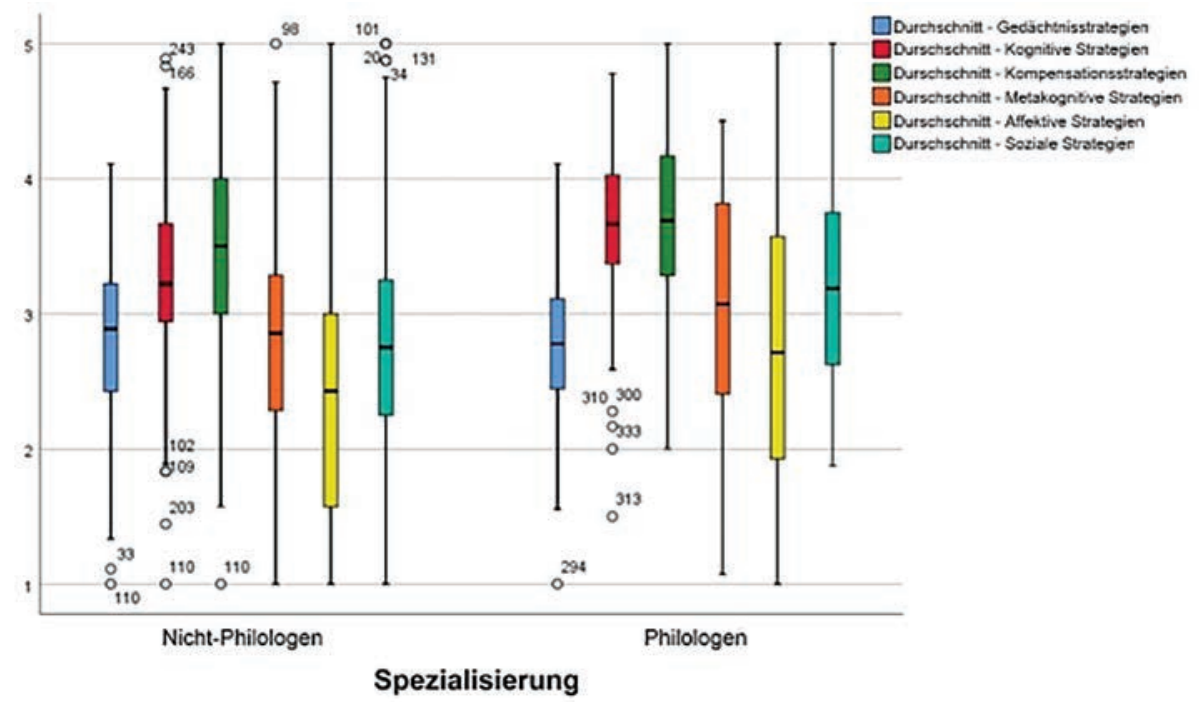

Graphik 2: Box-Plots der Durchschnittswerte von angewandten Strategien

5 Durchschnitt 
Aus dem graphisch dargestellten Vergleich ist zu sehen, dass die Anwendung von Gedächtnisstrategien bei Philologen und Nicht-Philologen ungefähr identisch ist. Der BoxPlot deutet ein höheres Maß im Gebrauch der restlichen Lernstrategien bei der Philologen-Gruppe im Vergleich mit der Gruppe der Nicht-Philologen an. Mit nachfolgenden Tests wurde überprüft, ob die Unterschiede statistisch signifikant sind. Im Hinblick auf die Größe der Nicht-Philologen-Gruppe und mit Hinblick auf die Datennormalität bei den Philologen wurde eine Überprüfung mittels eines $t$-Tests für zwei unabhängige Auswahlen durchgeführt. Für den Vergleich wurde auch der nichtparametrische MannWhitney-Test für zwei unabhängige Auswahlen angewendet. Die $t$-Test-Ergebnisse sind in Tabelle 3 zu sehen.

\begin{tabular}{|l|c|c|c|c|c|}
\hline & $\begin{array}{c}\text { Levene's Test } \\
\text { for Equality } \\
\text { of Variances }\end{array}$ & $\begin{array}{c}\text { t-test for } \\
\text { Equality of } \\
\text { Means }\end{array}$ & \multicolumn{3}{|c|}{ Mann-Whitney-Test } \\
\cline { 2 - 6 } & Sig. & Sig. (2-tailed) & $\mathbf{U}$ & $\mathbf{Z}$ & $\begin{array}{c}\text { Asymp. Sig. } \\
\text { (2-tailed) }\end{array}$ \\
\hline Gedächtnisstrategien & 0,329 & 0,467 & 9621,500 &,- 817 &, 414 \\
\hline Kognitive Strategien & 0,536 & 0,000 & 6293,500 & $-5,041$ &, 000 \\
\hline Kompensationsstrat. & 0,143 & 0,028 & 8480,000 & $-2,161$ &, 031 \\
\hline Metakognitive Strategien & 0,098 & 0,004 & 7853,000 & $-2,855$ &, 004 \\
\hline Affektive Strategien & 0,550 & 0,010 & 8043,500 & $-2,493$ &, 013 \\
\hline Soziale Strategien & 0,553 & 0,001 & 7255,500 & $-3,494$ &, 000 \\
\hline
\end{tabular}

Tabelle 3: Teststatistiken für die Gleichheit einzelner Lernstrategien zwischen der Philologenund Nicht-Philologen-Gruppe

In der Tabelle ist zuerst das Ergebnis des Levene-Tests zur Ermittlung der Gleichheit der Streuungen zwischen der Philologen-Gruppe und der Nicht-Philologen-Gruppe für die jeweilige Lernstrategie dargestellt. Es ist ersichtlich, dass alle $p$-Werte höher als 0,05 sind. Deshalb ist die Voraussetzung für eine Streuungsübereinstimmung nicht widerlegt, und dies gilt für alle Lernstrategien. Weiter wurde ein $t$-Test über die Durchschnittsübereinstimmung für zwei unabhängige Gruppen mit gleicher Streuung durchgeführt. Die $p$-Werte des Tests sind in der dritten Spalte der Tabelle 3 angegeben. Für die Gedächtnisstrategie ergab sich ein $p$-Wert von 0,467 . Aus diesem Grund ist es nicht möglich, die Hypothese der Gleichheit der Mittelwerte zu widerlegen. Bei anderen Lernstrategien ergaben sich entsprechende $p$-Werte des t-Tests in einem Ausmaß, das kleiner als 0,005 ist. Deshalb ist auf dem $5 \%$-Signifikanzniveau die Hypothese der Mittelwertegleichheit zwischen der Philologen-Gruppe und der Nicht-Philologen-Gruppe widerlegt. In den weiteren Spalten der Tabelle 4 sind die erhobenen Teststatistiken und die $p$-Werte des Mann-Whitney-Tests angeführt, die die Ergebnisse des t-Tests bestätigen.

Die Tabelle 5 präsentiert ausgewählte statistische Charakteristiken, die das Anwendungsmaß von Lernstrategien sowohl bei der Philologen-Gruppe als auch bei der Gruppe der Nicht-Philologen beschreiben. Bei den Gedächtnisstrategien der Nicht-Philologen-Gruppe ergab sich ein höherer Durchschnitt und ein höherer Median, dieser Unterschied ist jedoch statistisch nicht signifikant. Bei anderen Lernstrategien sind der 
Durchschnitt und der Median bei der Philologen-Gruppe höher, und die Tests haben diese Unterschiede als statistisch signifikant bestätigt.

\begin{tabular}{|l|l|c|c|c|c|c|c|}
\hline & $\begin{array}{c}\text { Spezialisie- } \\
\text { rung }\end{array}$ & $\begin{array}{c}\text { Gedächt- } \\
\text { nis-strate- } \\
\text { gien }\end{array}$ & $\begin{array}{c}\text { Kognitive } \\
\text { Strat. }\end{array}$ & $\begin{array}{c}\text { Kompensa- } \\
\text { tionsstrate- } \\
\text { gien }\end{array}$ & $\begin{array}{c}\text { Metakogn. } \\
\text { Strat. }\end{array}$ & $\begin{array}{c}\text { Affekti- } \\
\text { ve Strat. }\end{array}$ & $\begin{array}{c}\text { Soziale } \\
\text { Strat. }\end{array}$ \\
\hline \multirow{2}{*}{ Durchschnitt } & Nicht-Phil. & 2,830 & 3,270 & 3,487 & 2,827 & 2,413 & 2,796 \\
\cline { 2 - 8 } & Philologen & 2,766 & 3,648 & 3,705 & 3,108 & 2,745 & 3,168 \\
\hline \multirow{2}{*}{ Median } & Nicht-Phil. & 2,889 & 3,222 & 3,500 & 2,857 & 2,429 & 2,750 \\
\cline { 2 - 8 } & Philolgen & 2,778 & 3,667 & 3,690 & 3,071 & 2,714 & 3,188 \\
\hline $\begin{array}{l}\text { Standard- } \\
\text { abweichung }\end{array}$ & Nicht-Phil. & 0,625 & 0,628 & 0,685 & 0,738 & 0,967 & 0,820 \\
\cline { 2 - 8 } & Philologen & 0,612 & 0,620 & 0,602 & 0,851 & 1,018 & 0,766 \\
\hline
\end{tabular}

Tabelle 5: Statistische Charakteristiken der Durchschnitte von Lernstrategien

Ergebnis: Die Daten haben keinen Unterschied im Anwendungsmaß von Gedächtnisstrategien zwischen der Philologen- und der Nicht-Philologen-Gruppe ergeben. Die von uns erhobenen Daten zeigen jedoch ein höheres Anwendungsmaß von kognitiven, metakognitiven, affektiven und sozialen Strategien sowie von Kompensationsstrategien zugunsten der Philologen-Gruppe im Vergleich mit der Nicht-Philologen-Gruppe.

\section{Diskussion}

Auf Grund der gesamten Analyse unserer Forschung hat sich ergeben, dass beide Gruppen unserer Probanden, sowohl Philologen als auch Nicht-Philologen, im Wesentlichen identische Lernstrategien anwenden, jedoch in unterschiedlichem Maß. Das bedeutet also, dass wir unsere erste Hypothese, in der wir vorausgesetzt haben, dass Studierende philologisch orientierter Studienfächer mehr Lernstrategien anwenden, als es bei Studierenden nicht-philologisch orientierter Studienfächer der Fall ist, falsifiziert konnten.

Nicht ganz problematisch bleibt dabei jedoch die Frage, ob diese Anwendung bewusst oder unbewusst ist. Wie es sich laut der Antworten unserer Probanden gezeigt hat, setzen viele von ihnen die einzelnen Lernstrategien eher spontan ein, ohne mit ihnen vorher bekannt gemacht zu werden.

Bei der zweiten Hypothese, in der wir erwarteten, dass es gravierende Unterschiede in den angewandten Lernstrategien zwischen Studierenden der philologisch orientierten und nicht-philologisch orientierten Studienfächer gibt, haben wir keinen markanten Unterschied im Anwendungsmaß von Gedächtnisstrategien zwischen beiden Gruppen der Probanden identifiziert. Auf der anderen Seite zeigte sich jedoch ein höheres Anwendungsmaß von kognitiven, metakognitiven, affektiven und sozialen Strategien sowie von Kompensationsstrategien zugunsten der Philologen-Gruppe. Diese Hypothese mussten wir auf Grunde der erhobenen Daten teilweise revidieren und zwar im Bereich der Gedächtnisstrategien. Bei den restlichen Strategien (kognitive, metakognitive, affektive, soziale) hat sich unsere Hypothese bestätigt. 
Wir plädieren darum dafür, dass Studierende mit einzelnen Typen von Lernstrategien im Rahmen des Fremdsprachenunterrichts explizit vertraut gemacht werden sollten, denn die Kenntnis dieser Lernstrategien kann ihnen das Lernen nicht nur einer Fremdsprache leichter machen.

\section{Schlusswort}

Der Beitrag stellte Teilergebnisse einer breit angelegten Studie zum Einsatz von Lernstrategien beim Aneignen von Deutsch seitens tschechischer Universitäts- und Hochschulstudierender dar. Die Autoren haben sich dabei insb. auf die Unterschiede in der Zahl und dem Anwendungsmaß der angewandten Lernstrategien zwischen philologisch orientierten und nicht-philologisch orientierten Studierenden konzentriert. Es wurde festgestellt, dass die Zahl der angewandten Lernstrategien bei Philologen höher ist als bei Nicht-Philologen und dass Philologen fast alle Strategien (außer Gedächtnisstrategien) öfter einsetzen als die Nicht-Philologen. Dabei können wir uns jedoch die Frage stellen, warum dem so ist? Ist der Grund dafür, dass die Zahl der eingesetzten Strategien bei Philologen höher ist, die Tatsache, dass diese Gruppe der Probanden in einem intensiveren Kontakt mit der Sprache ist, so dass sie, obwohl sie vielleicht mit den einzelnen Lernstrategien nicht explizit bekannt gemacht worden sind, deswegen den Algorithmus einzelner Lernstrategien selbst finden? Oder sind dabei noch andere Variablen im Spiel? Diese Fragen könnten ein Aufruf für weitere Forschungen auf dem Gebiet der Lehrstrategien sein. Im Zusammenhang damit sind wir der Meinung, dass es empfehlenswert wäre, den einzelnen Lernstrategien im eigenen Fremdsprachenunterricht mehr Aufmerksamkeit zu widmen, damit unseren Schüler*innen/Studierenden die Nützlichkeit, strategisch zu lernen, stärker bewusst wird.

\section{Literaturverzeichnis}

Bialystok, Ellen (1981): The Role of Conscious Strategies in Second Language Proficiency. In: The Modern Language Journal 65/1981, S. 24-35.

Bimmel, Peter/Rampillon, Ute (2000): Lernerautonomie und Lernstrategien. München: Langenscheidt.

Cohen, Andrew D. (1990): Strategies in Second Language Learning. Insights from Research. In: Foreign/second language pedagogy Research. A Commemorative Volume for Claus Faerch. Hrsg. v. Robert Phillipson, Eric Kellermann, Larry Selinker, Michael Sharwood-Smith u. Merrill Swain. Clevedon: Multilingual Matters Lt.d., S. 107-119.

Cohen, Andrew D. (2014): Strategies in learning and using a second language. 2. ed. London/ New York: Routledge.

Ellis, Rod (1994): The study of second language acquisition. Oxford: Oxford University Press. Entwistle, Noel/Ramsden, Paul (1983): Understanding Student Learning. London: Croom Helm, S. 6-28. 
Færch, Claus /Kasper, Gabriele (1983): On identifying commucation strategies in inter-language production. In: Strategies in interlanguage communication. Hrsg. v. Claus Færch u. Gabriele Kasper. New York: Longman, S. 210-238.

Friedrich, Helmut Felix/Mandl, Heinz (1992): Lern- und Denkstrategien - ein Problemaufriß. In: Lern- und Denkstrategien. Analyse und Intervention. Hrsg. v. Heinz Mandl u. Helmut Felix Friedrich. Göttingen: Hogrefe, S. 3-54.

Janíková, Věra (2007): Autonomní učení a lexikální strategie při osvojování cizích jazyků: Teoretická východiska. Doporučení pro praxi. Styly a strategie učení. Lexikální strategie v teorii a praxi. Brno: Masarykova univerzita.

Jamieson-Noel, Dianne/Winne, Philip H. (2003): Comparing Self-Reports to Traces of Studying Behavioras Representations of Students' Studying and Achievement. In: Zeitschrift für pädagogische Psychologie 17/2003, S. 159-171.

Knowles, Malcolm (1995): Self-directed Learning. A Guide for Learners and Teachers. Chicago: Follett Publ. Comp.

Lojová, Gabriela/Vlčková, Kateřina (2011): Styly a strategie učení ve výuce cizích jazyků. Vyd. 1. Praha: Portál.

Lompscher, Joachim (1996): Erfassung der Lernstrategien auf der Reflexionsebene. In: LLF-Berichte 13/1996, S. 10-43.

Marton, Ference/Säljö, Roger (1976): On qualitative Differences in Learning: Outcome and Process. In: British Journal of Educational Psychology 46/1976, S. 4-11.

Netolická, Veronika (2008): Testy normality. Přírodovědecká fakulta Univerzity Palackého v Olomouci : Katedra matematické analýzy a aplikací matematiky.

O’Malley, Michael J./Chamot, Anna U. (1990): Learning Strategies in Second Language Acquisition. Cambridge: Cambridge University Press.

Oxford, Rebecca L. (1990): Language learning Strategies. What Every Teacher Should Know. Boston: Heinle \& Heinle Publishers.

Rampillon, Ute (1985): Lerntechniken im Fremdsprachenunterricht. München: Hueber.

Schiefele, Ulrich/Streblow, Lilian/Ermgassen, Ulrich/Moschner, Barbara (2003): Lernmotivation und Lernstrategien als Bedingungen der Studienleistung: Ergebnisse einer Längsschnittstudie. In: Zeitschrift für Pädagogische Psychologie 17/2003, S. 185-198.

Streblow, Lilian/ Schiefele, Ulrich (2006): Lernstrategien im Studium. In: Handbuch Lernstrategien. Hrsg. v. Heinz Mandl u. Helmut Felix Friedrich:. Göttingen: Hogrefe, S. 352-364.

Tarone, Ellen (1983): Some Thoughts on the Notion of Communication Strategie. In: Strategies in Interlinguale Communication. Hrsg. v. Claus Færch u. Gabriele Kasper. London: Longman, S. 61-74.

Tarone, Ellen/Cohen, Andrew D./Dumas, Guy (1983): A closer look at some interlanguage terminology: a framework for communication strategie. Reprint in: Strategies in interlanguage communication. Hrsg. v. Claus Færch u. Gabriele Kasper. New York: Longman, S. 4-14.

Vlčková, Kateřina (2007): Strategie učení cizímu jazyku. Výsledky výzkumu používání strategiî a jejich efektivity na gymnáziích. Brno: Paido.

Weinstein, Claire E./Mayer, Richard E. (1986): The teaching of learning strategies. In: Handbook of research on teaching. Hrsg. v. Merlin Wittrock. New York: Macmillan, S. 315-327.

Wenden, Anita L. (1987): Conceptual background and utility. In: Learner strategies in language learning. Hrsg. v. Anita Wenden u. Joan Rubin. New York u. a.: Prentice/Hall International, S. 3-13.

Westhof, Gerhard, J. (2001): Zweitsprachenerwerb als Lernaktivität II: Lernstrategien - Kommunikationsstrategien - Lerntechniken. In: Deutsch als Fremdsprache: ein internationales Handbuch. Hrsg. v. Gerhard Helbig et al. Berlin: de Gruyter.

Wild, Klaus-Peter (2000): Lernstrategien im Studium. Münster: Waxmann Verlag. 
Doc. PhDr. Martin Lachout, Ph.D. / Martin.Lachout@ujep.cz

Univerzita Jana Evangelisty Purkyně, Filozofická fakulta, Katedra germanistiky Pasteurova 13, 40001 Ústí nad Labem, CZ

Mgr. Ondřej Moc, Ph.D. / ondrej.moc@ujep.cz

Univerzita Jana Evangelisty Purkyně, Fakulta sociálně ekonomická, Katedra matematiky a informatiky, Moskevská 54, 40096 Ústí nad Labem, CZ

\section{Mgr. Eva Krovová}

Obchodní akademie

Ústí nad Labem, CZ 
